\title{
Evaluation of the learning curve of transanal total mesorectal excision: single-centre experience
}

\author{
Mateusz Rubinkiewicz ${ }^{1}$, Katarzyna Truszkiewicz ${ }^{1}$, Michał Wysocki ${ }^{1,2}$, Jan Witowski ${ }^{1}$, Grzegorz Torbicz ${ }^{1}$, \\ Michal M. Nowakowski ${ }^{1}$, Andrzej Budzynski ${ }^{1,2}$, Michał Pędziwiatr ${ }^{1,2}$ \\ ${ }^{1} 2^{\text {nd }}$ Department of General Surgery, Jagiellonian University Medical College, Krakow, Poland \\ ${ }^{2}$ Centre for Research, Training and Innovation in Surgery (CERTAIN Surgery), Krakow, Poland
}

\begin{abstract}
Introduction: Transanal total mesorectal excision (TATME) has been recently proposed to overcome the difficulties of the standard TME approach, allowing better visualization and dissection of the mesorectal fascia. Although TaTME seems very promising, the evidence and body of knowledge on achieving proficiency in performing it are still sparse. Aim: To evaluate the learning curve of TaTME based on a single centre's experience.

Material and methods: Consecutive patients undergoing TaTME since 2014 in a tertiary referral department were included in the study. All procedures were performed by one experienced surgeon. CUSUM curve analyses were performed to evaluate learning curves.

Results: Sixty-six patients underwent TaTME. After analysis of postoperative morbidity rate, intraoperative adverse effects and operative time, we estimated that 40 cases are needed to achieve TaTME proficiency. Subsequently, patients were divided into two groups: before (40 patients) and after overcoming the learning curve (26 patients). Group 1 had higher readmission $(p=0.041)$ and complication rates $(p=0.019)$. There were no statistically significant differences in terms of intraoperative adverse effects, length of stay or pathological quality of the specimen.

Conclusions: Transanal total mesorectal excision is a promising yet technically demanding procedure and requires at least 40 cases to complete the learning curve. More data are needed to introduce it as a standard procedure for low rectal cancer treatment.
\end{abstract}

Key words: transanal total mesorectal excision, total mesorectal excision, rectal cancer, learning curve.

\section{Introduction}

Total mesorectal excision (TME) is currently the standard surgical approach in the treatment of rectal cancer $[1,2]$. Despite progress in the technique, surgery for low rectal cancer remains technically challenging. Moreover, according to several well-designed randomized controlled trials, introduction of minimally invasive techniques, although beneficial in terms of short-term outcomes, did not lead to significantly improved oncological outcomes [1, 3, 4].
Improvement of the results can be expected only by raising the quality of the surgical technique of mesorectal excision. The final result can be easily assessed by pathological evaluation of the resected specimen [5].

To overcome intraoperative difficulties of TME performed by the transabdominal approach (both open and laparoscopic), transanal total mesorectal excision (TaTME) has been proposed [6]. This promising technique improves visualization and dissection of the mesorectal fascia plane, especially in obese 
patients or in a narrow, irradiated pelvis. It is expected that this will result in more precise and atraumatic dissection, improving quality of the operative specimen and reducing positive resection margins [6-9].

It has been shown that the annual volume of the surgical centre and the team's experience are crucial to achieve good oncological outcomes in rectal cancer treatment $[10,11]$. To achieve a high level of proficiency, surgeons must go through the learning curve that is estimated to require at least 50 cases of laparoscopic low anterior resection [12, 13]. Since TATME is a relatively new technique, the number of operations that should be performed to reach a stable level of skill is still poorly defined. Thus, we designed a study to evaluate the learning curve of TaTME based on our experience.

\section{Aim}

The aim of the study was to evaluate the learning curve of TaTME based on a single centre's experience.

\section{Material and methods}

\section{Study design}

We performed a retrospective analysis of a prospectively collected database. Consecutive patients operated on with TaTME technique were included in the study. We are a tertiary referral department in a university hospital with an annual volume of at least 50 rectal resections. Transanal total mesorectal excision was introduced in 2014 as a procedure for low rectal cancer ( $<5 \mathrm{~cm}$ from the anal verge). Our department is a participant in the COLOR III trial [14]. In this study we analysed a single surgeon's experience. In all presented cases the procedures were performed by the same experienced surgeon.

Every patient underwent diagnostic colonoscopy with tumour biopsy and subsequent pathological confirmation of adenocarcinoma. All patients had pelvic magnetic resonance imaging (MRI) and thoracic and abdominal computed tomography (CT) scan evaluation as a routine preoperative workup. Patients classified as stage T3 or N+ in MRI were submitted to neoadjuvant treatment. In case of suspicion of threatened circumferential resection margin (CRM) or infiltration of the internal anal sphincter, $M R I$ was repeated after completion of chemoradiotherapy.
Before implementation of TaTME, the surgical team underwent training in reference centres that included cadaver-based hands-on training. The operative technique is described elsewhere [6]. As a modification of the original technique, we use the TEO TEM platform by Karl Storz. The procedure is routinely performed in a one-team approach. The anastomosis is performed with a circular stapler or alternatively hand-sewn if stapled anastomosis is technically not feasible. The enhanced recovery after surgery (ERAS) protocol with good compliance was used in perioperative care in all cases [15-17].

The surgical specimen was assessed by an experienced pathologist. The quality of mesorectal excision was evaluated in accordance with Quirke criteria [18].

For the purpose of the study we performed CUSUM analysis of postoperative morbidity, intraoperative adverse effects, operative time and quality of resected specimen. Based on CUSUM curves we divided the patients into two groups: group 1 consisted of patients operated on during the learning curve and group 2 patients operated on after reaching the plateau of the learning curve.

\section{Statistical analysis}

All data were analysed with StatSoft Statistica version 13.0 PL (StatSoft Inc., Tulsa, OK, USA). The results are presented as mean \pm standard deviation (SD), median and interquartile range, and odds ratios (ORs) with 95\% confidence intervals (Cls) when appropriate. To assess statistical significance of qualitative data differences between subgroups, Pearson's $\chi^{2}$ test and discriminant analysis were used. CUSUM curve analyses were used to estimate cut-off values in terms of the number of procedures performed to reach stabilisation of the learning curve. Results were considered statistically significant when the $p$-value was less than 0.05 .

\section{Results}

\section{Patients}

We included 66 patients who underwent TaTME performed by the same surgeon. Forty-four (66.7\%) patients were male. Median age was 64 (56-71) years. Fifty-four (81.82\%) patients required neoadjuvant treatment. Eighteen (28.5\%) patients had stage 0 according to the AJCC classification, 16 (23.8\%) 
stage I, 14 (17.5\%) stage II, 16 (4.8\%) stage III and $2(3.2 \%)$ stage IV. The characteristics of groups are presented in Table I.

\section{Analysis of the CUSUM charts}

We observed a drop in the postoperative morbidity rate after the $30^{\text {th }}$ case and in intraoperative adverse effects after the $35^{\text {th }}$ case (Figures 1 and 2). We also observed stabilization in the op- erating time at the $40^{\text {th }}$ case (Figure 3). After that, we observed a slight descent of the curve. We did not identify changes in the learning curve regarding pathological quality of the specimen (Figure 4). Based on those analyses, we estimated that at least 40 surgery cases are needed to stabilize the learning curve. Therefore, we divided our patients into two groups: group 1 (first 40 cases) and group 2 (remaining patients).

Table I. Characteristics of study groups

\begin{tabular}{|lcccc|}
\hline Parameter & $\begin{array}{c}\text { All patients } \\
(N=66)\end{array}$ & $\begin{array}{c}\text { Group 1 } \\
(n=40)\end{array}$ & $\begin{array}{c}\text { Group 2 } \\
(n=26)\end{array}$ & $P$-value \\
\hline Male & $44(66.7 \%)$ & $31(78 \%)$ & $13(50 \%)$ & 0.29 \\
\hline Age, median (IQR) [years] & $64(56-71)$ & $62.5(54.5-72.5)$ & $65(57-71)$ & 0.78 \\
\hline BMI, median (IQR) [kg/m²] & $25.62(23.15-29.35)$ & $25.5(23.7-29.0)$ & $25.9(21.9-29.7)$ & 0.65 \\
\hline Depth from anal verge, Median (IQR) [cm] & $3(2-4)$ & $3(2-4)$ & $4(2-5)$ & 0.08 \\
\hline Preoperative radiotherapy & $54(81.82 \%)$ & $35(88 \%)$ & $19(73 \%)$ & 0.63 \\
\hline History of previous surgery & $15(22 \%)$ & $9(23 \%)$ & $7(27 \%)$ & 0.74 \\
\hline Operative time, median (IQR) [min] & $240(210-280)$ & $270(240-300)$ & $210(170-240)$ & 0.0002 \\
\hline Blood loss, median (IQR) [ml] & $100(50-200)$ & $150(50-200)$ & $50(50-150)$ & 0.006 \\
\hline Diverting ileostomy & $57(86 \%)$ & $15(100 \%)$ & $15(100 \%)$ & 0.001 \\
\hline Intraoperative adverse events & $6(9.09 \%)$ & $5(20 \%)$ & $1(13 \%)$ & 0.03 \\
\hline Postoperative morbidity & $15(22.73 \%)$ & $13(33 \%)$ & $2(8 \%)$ & 0.001 \\
\hline Clavien-Dindo III-V & $7(10.6 \%)$ & $6(15 \%)$ & $1(4 \%)$ & 0.30 \\
\hline LOS, median (IQR) [days] & $5(4-7)$ & $6(4-8)$ & $5(4-6)$ & 0.375 \\
\hline Readmissions & $8(12.12 \%)$ & $7(18 \%)$ & $1(4 \%)$ & 0.04 \\
\hline
\end{tabular}

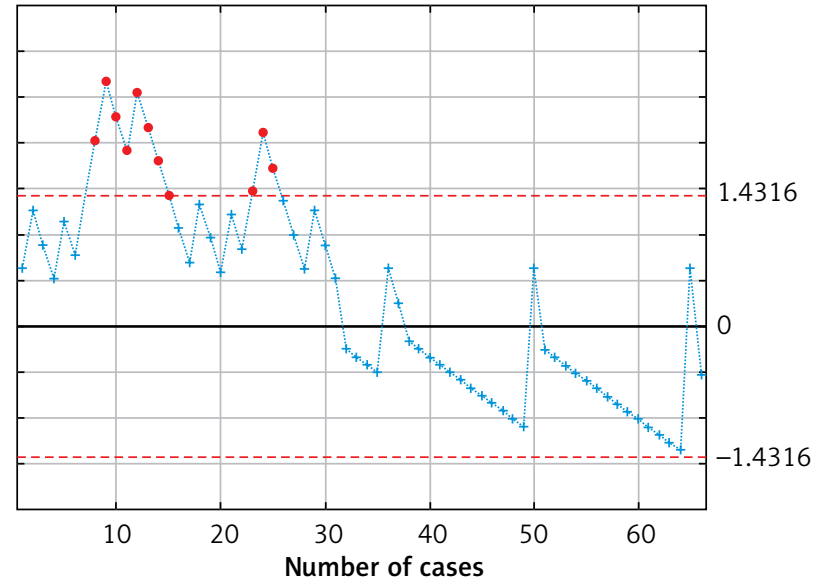

Figure 1. CUSUM graph for postoperative morbidity

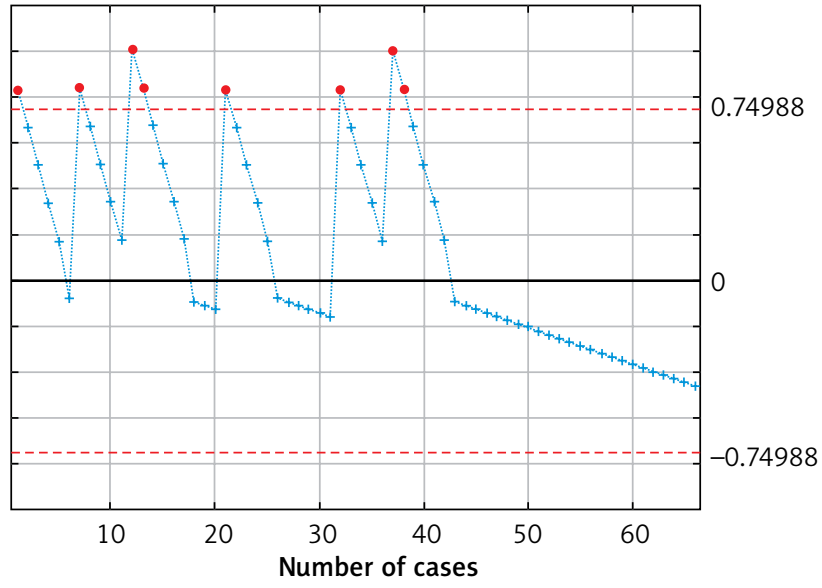

Figure 2. CUSUM graph for intraoperative adverse effects 


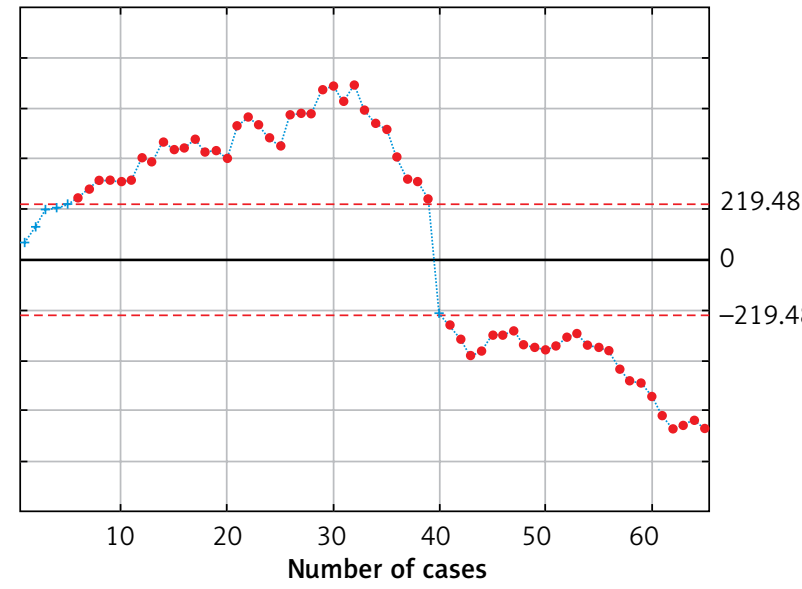

Figure 3. CUSUM graph for operative time

\section{Sub-group analysis}

Median overall operative time was 240 (IQR: 210-280) min. In total, 6 intraoperative adverse events were noted (4 purse string failures, 1 inability to maintain stable insufflation of the working space in the pelvis and 1 case of bleeding in the perineal step of the procedure). There were no significant differences between the groups $(p=0.216)$.

Postoperative complications occurred in $22.73 \%$ of patients (Table II). There were significant differences between the groups $(p=0.019)$ - a majority of complications occurred within first 30 cases $(12 / 15,80 \%)$.

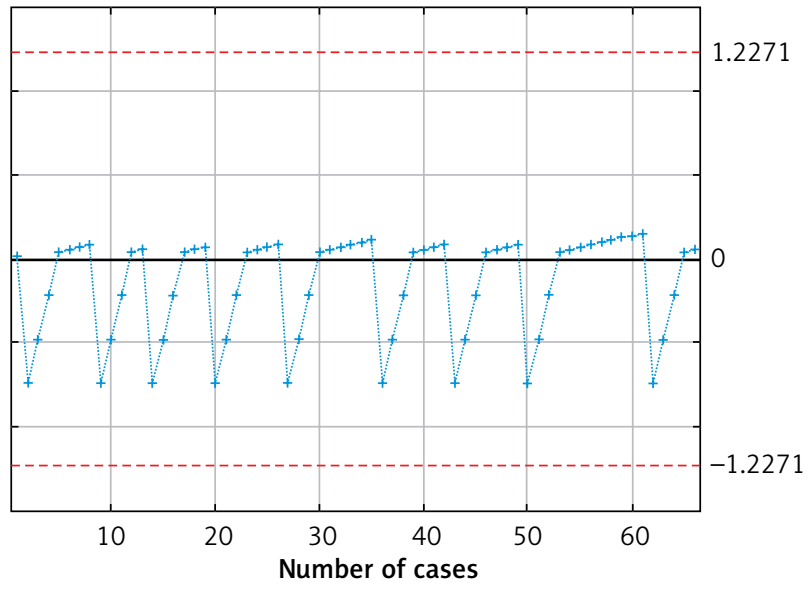

Figure 4. CUSUM graph for quality of resected specimen

Pathological outcomes were stable during the learning process (Table III). One patient in group 1 had positive CRM and 1 patient in group 2 had positive distal resection margin (DRM). Median CRM and DRM did not differ between the groups ( $p=0.542$ and $p=0.573$, respectively). There were no differences regarding pathological quality of the specimen.

We observed a significant difference in the readmission rate $(p=0.041)$. Most of the readmissions occurred in group 1 (63\%). The median overall hospital length of stay (LOS) was 5 days and was not statistically different between the groups

Table II. Number of complications

\begin{tabular}{|c|c|c|c|c|}
\hline Clavien-Dindo Grade & Group $1(N=40)$ & $N(\%)$ & Group $2(N=26)$ & $N(\%)$ \\
\hline V & & & & 0 \\
\hline IV & $\begin{array}{l}\text { Anastomotic leakage with concomitant } \\
\text { sepsis (operative treatment) }\end{array}$ & $1(2.5)$ & & 0 \\
\hline IIIb & $\begin{array}{l}\text { Anastomotic leakage (operative treat- } \\
\text { ment) } \\
\text { Postoperative ileus (operative treat- } \\
\text { ment) }\end{array}$ & $\begin{array}{l}3(7.5) \\
1(2.5)\end{array}$ & Anastomotic leakage & $1(4)$ \\
\hline Illa & $\begin{array}{c}\text { Anastomotic leakage (percutaneous } \\
\text { drainage of the near-anastomotic } \\
\text { abscess) }\end{array}$ & $1(2.5)$ & & 0 \\
\hline ॥ & $\begin{array}{c}\text { High output stoma } \\
\text { Anastomotic leakage (conservative } \\
\text { treatment) }\end{array}$ & $\begin{array}{l}2(5) \\
2(5)\end{array}$ & & 0 \\
\hline I & $\begin{array}{l}\text { Postoperative fever of unknown origin } \\
\text { Surgical site infection } \\
\text { Radial nerve paresis (due to prolonged } \\
\text { compression on the operating table) }\end{array}$ & $\begin{array}{l}1(2.5) \\
1(2.5) \\
1(2.5)\end{array}$ & Wound hematoma & $1(4)$ \\
\hline Total & & $13(33)$ & & $2(8)$ \\
\hline
\end{tabular}


Table III. Pathological outcomes

\begin{tabular}{|c|c|c|c|c|c|}
\hline \multicolumn{2}{|c|}{ Parameter } & \multirow{2}{*}{$\begin{array}{c}\text { All patients } \\
(N=66) \\
18(28.57 \%)\end{array}$} & \multirow{2}{*}{$\begin{array}{c}\text { Group } 1 \\
(N=40)\end{array}$} & \multirow{2}{*}{$\begin{array}{c}\text { Group } 2 \\
(N=26)\end{array}$} & \multirow{2}{*}{$\begin{array}{c}P \text {-value } \\
0.242\end{array}$} \\
\hline AJCC & 0 & & & & \\
\hline & 1 & $16(23.81 \%)$ & 8 & 8 & \\
\hline & $\|$ & $14(17.46 \%)$ & 10 & 4 & \\
\hline & III & $16(4.76 \%)$ & 6 & 10 & \\
\hline & IV & $2(3.17 \%)$ & 1 & 1 & \\
\hline \multicolumn{2}{|c|}{ Distal margin, median (IQR) [mm] } & $10.5(7.5-15.5)$ & $15(10-17)$ & $10(6-15)$ & 0.14 \\
\hline \multicolumn{2}{|c|}{ Radial margin, median (IQR) [mm] } & $9.25(2.9-13.5)$ & $9.5(2.9-13.5)$ & $9.25(5-16)$ & 0.872 \\
\hline \multicolumn{2}{|c|}{$\begin{array}{l}\text { Quality of mesorectal excision } \\
\text { Complete/Nearly complete }\end{array}$} & $57(86 \%) / 9(14 \%)$ & $34(85 \%) / 6(15 \%)$ & $23(89 \%) / 3(11 \%)$ & 0.91 \\
\hline
\end{tabular}

$(p=0.375)$. However, median LOS in group 1 was longer by one day (6 (4-12)).

\section{Discussion}

This is one of the first studies evaluating the learning curve of TaTME. We found that a significant drop of perioperative complications and operative time happens after the first 40 cases.

Available data about TaTME are sparse and come from large, high-volume centres, which specialize in laparoscopic surgery $[19,20]$. Moreover, the definition of TaTME is not unified and there are several approaches that differ substantially yet still are called TaTME. The St. Gallen consensus on TaTME implementation delivered a standard technique, which is included as part of the COLOR III protocol $[14,21]$. Nonetheless, available studies are heterogeneous regarding both operative technique and patient population. This creates a bias as in the case of the meta-analysis by Hu et al. that included studies assessing different transanal approaches that do not necessarily follow the principles of TaTME [22]. Moreover, almost all currently available studies comparing TaTME to other techniques present data from the middle of the learning curve $[23,24]$.

Transanal total mesorectal excision is a complex endoscopic procedure that requires a learning curve to obtain stable results. There are several factors that can shorten the learning process, including experience in laparoscopic technique or participation in hands-on cadaver-based courses [21]. It is also a possible explanation of the shorter learning curve in our institution than reported before $[25,26]$.
Perhaps the most clinically significant parameters taken into consideration when analysing the learning curve are postoperative complications. We observed a drop and stabilisation of the learning curve regarding postoperative complications after the $30^{\text {th }}$ case. Available studies show comparable results of TaTME to laparoscopic TME (LATME) [9, $27,28]$. However, most of these studies are based on small data samples from the initial period of the TaTME learning curve, whereas LaTME cases are usually matched from larger databases [27, 28]. In large datasets overall morbidity of LaTME is estimated to be around 36\% [1]. Lacy et al. reported overall morbidity of $24 \%$ with major complications (Clavien-Dindo $\mathrm{III}-\mathrm{V}$ ) reaching the $10 \%$ rate after 140 TaTME cases [29]. Moreover, Koedam reported a rate of $17.5 \%$ for overall complications after the first 40 cases, which was defined as a cut-off for the learning curve. This trend was not observed by Lee et al., who reported a constant rate of postoperative complications in the range between $42 \%$ and $45 \%$. We observed a significant drop in overall morbidity, which allowed us to define a period after learning curve stabilization. During the first period we used hand-sewn or stapled anastomosis, depending on its distance from the anal verge. However, further experiences allowed us to use stapled anastomosis in almost every case, which resulted in a drop of the anastomotic leakage rate.

One of the main problems that might occur while learning TaTME technique is specific intraoperative adverse effects (IAE), which - due to differences in the technique - do not occur in LaTME. In our mate- 
rial, the most common IAE was purse string failure. In theory, this may result in bowel content spillage and contamination of the operative field, including with cancer cells [30]. Other authors also point out that perforation of the bowel wall or gas embolism may occur [19, 31]. Mege et al. revealed that IAE in TaTME are more common than in LaTME, and may also present as urethral injury, bladder perforation or vagina wall damage [27]. A narrow pelvis and difficult anatomy after radiotherapy may also lead to iliac vessel injury [26]. Again, in our material the majority of IAE occurred within the first 35 cases. Lee et al. observed a similar drop from $12 \%$ to $6 \%$, although the number of cases required for passing the learning curve was estimated at 51 TaTME procedures [26].

Operative time is probably the most frequently used parameter for evaluation of the learning curve, although it plays a limited role in clinical evaluation. CUSUM analysis revealed its stabilisation after 40 cases from a median of 270 to $210 \mathrm{~min}$. Other authors report an even more significant drop, but it is frequently associated with implementation of a two-team approach [29]. Koedam et al. observed a substantial reduction in the operative time after introduction of the two-team approach. However, both in one- and two-team approaches the operating time did not change significantly with surgical experience [25]. Lee et al. observed shortening of the operative time after the $36^{\text {th }}$ case, although they did not indicate the moment of switching the technique to the two-team approach [26].

Our study has some considerable limitations. Firstly, the study group consists of 66 cases, which limits the power of statistical tests. However, we observed stabilisation of operative parameters after the $40^{\text {th }}$ case, so our material could be considered sufficient. We also evaluated the learning curve of a single surgeon, who is already an expert in minimally invasive techniques. More cases are required to evaluate the learning process in the entire department. In this study we also did not analyse functional and long-term outcomes, which are crucial for patients. Nonetheless, TaTME is still under initial evaluation and none of the studies published in the literature assessed long-term outcomes. We expect such studies in the next few years, as TaTME was introduced in 2010 and long-term observations should become available shortly [6]. They are also one of the crucial points of COLOR III - a ran- domized multi-centre study comparing TaTME with LaTME [14].

\section{Conclusions}

Transanal total mesorectal excision is a promising procedure, but the technique is technically demanding and requires at least 40 cases to finish the learning curve. Having said that, more data are needed to evaluate TaTME and introduce it as a standard procedure for low rectal cancer treatment.

\section{Conflict of interest}

The authors declare no conflict of interest.

\section{References}

1. Małczak P, Mizera M, Torbicz G, et al. Is the laparoscopic approach for rectal cancer superior to open surgery? A systematic review and meta-analysis on short-term surgical outcomes. Videosurgery Miniinv 2018; 13: 129-40.

2. Nowakowski MM, Rubinkiewicz M, Gajewska N, et al. Defunctioning ileostomy and mechanical bowel preparation may contribute to development of low anterior resection syndrome. Videosurgery Miniinv 2018; 13: 306-14.

3. Abbas SK, Yelika SB, You K, et al. Rectal cancer should not be resected laparoscopically: the rationale and the data. Tech Coloproctol 2017; 21: 237-40.

4. van der Pas MH, Haglind E, Cuesta MA, et al. Laparoscopic versus open surgery for rectal cancer (COLOR II): short-term outcomes of a randomised, phase 3 trial. Lancet Oncol 2013; 14: 210-8.

5. Kitz J, Fokas E, Beissbarth T, et al. Association of plane of total mesorectal excision with prognosis of rectal cancer: secondary analysis of the CAO/ARO/AIO-04 phase 3 randomized clinical trial. JAMA Surg 2018; 153: e181607.

6. Sylla P, Rattner DW, Delgado S, Lacy AM. NOTES transanal rectal cancer resection using transanal endoscopic microsurgery and laparoscopic assistance. Surg Endosc 2010; 24: 1205-10.

7. Chen WH, Kang L, Luo SL, et al. Transanal total mesorectal excision assisted by single-port laparoscopic surgery for low rectal cancer. Tech Coloproctol 2015; 19: 527-34.

8. Piątkowski J, Jackowski M, Szeliga J, Nowak M. Transanal total mesorectal excision (TATME) - preliminary findings. Videosurgery Miniinv 2015; 10: 495-8.

9. Rubinkiewicz M, Nowakowski M, Wierdak M, et al. Transanal total mesorectal excision for low rectal cancer: a case-matched study comparing TaTME versus standard laparoscopic TME. Cancer Manag Res 2018; 10: 5239-45.

10. Hohenberger W, Merkel S, Hermanek P. Volume and outcome in rectal cancer surgery: the importance of quality management. Int J Colorectal Dis 2013; 28: 197-206.

11. García-Granero E, Martí-Obiol R, Gómez-Barbadillo J, et al. Impact of surgeon organization and specialization in rectal cancer outcome. Colorectal Dis 2001; 3: 179-84. 
12. Bege T, Lelong B, Esterni B, et al. The learning curve for the laparoscopic approach to conservative mesorectal excision for rectal cancer: lessons drawn from a single institution's experience. Ann Surg 2010; 251: 249-53.

13. Tekkis PP, Senagore AJ, Delaney CP, Fazio VW. Evaluation of the learning curve in laparoscopic colorectal surgery: comparison of right-sided and left-sided resections. Ann Surg 2005; 242: 83-91.

14. Deijen CL, Velthuis S, Tsai A, et al. COLOR III: a multicentre randomised clinical trial comparing transanal TME versus laparoscopic TME for mid and low rectal cancer. Surg Endosc 2016; 30: 3210-5.

15. Kisielewski M, Rubinkiewicz M, Pedziwiatr M, et al. Are we ready for the ERAS protocol in colorectal surgery? Videosurgery Miniinv 2017; 12: 7-12.

16. Pisarska M, Pędziwiatr M, Major P, et al. Laparoscopic gastrectomy with enhanced recovery after surgery protocol: single-center experience. Med Sci Monit 2017; 23: 1421-7.

17. Pędziwiatr M, Pisarska M, Major P, et al. Laparoscopic colorectal cancer surgery combined with enhanced recovery after surgery protocol (ERAS) reduces the negative impact of sarcopenia on short-term outcomes. Eur J Surg Oncol 2016; 42: 779-87.

18. Quirke P, Morris E. Reporting colorectal cancer. Histopathology 2007; 50: 103-12.

19. Penna M, Hompes R, Arnold S, et al. Transanal total mesorectal excision. Ann Surg 2017; 266: 111-7.

20. Rubinkiewicz M, Czerwińska A, Zarzycki P, et al. Comparison of short-term clinical and pathological outcomes after transanal versus laparoscopic total mesorectal excision for low anterior rectal resection due to rectal cancer: a systematic review with meta-analysis. J Clin Med 2018; 7: pii: E448.

21. Adamina M, Buchs NC, Penna M, et al. St. Gallen consensus on safe implementation of transanal total mesorectal excision. Surg Endosc 2018; 32: 1091-103.

22. Hu D, Jin P, Hu L, et al. The application of transanal total mesorectal excision for patients with middle and low rectal cancer. Medicine (Baltimore) 2018; 97: e11410.

23. Jiang HP, Li YS, Wang B, et al. Pathological outcomes of transanal versus laparoscopic total mesorectal excision for rectal cancer: a systematic review with meta-analysis. Surg Endosc 2018; 32: $2632-42$.

24. Xu W, Xu Z, Cheng H, et al. Comparison of short-term clinical outcomes between transanal and laparoscopic total mesorectal excision for the treatment of mid and low rectal cancer: a meta-analysis. Eur J Surg Oncol 2016; 42: 1841-50.

25. Koedam TWA, Veltcamp Helbach M, van de Ven PM, et al. Transanal total mesorectal excision for rectal cancer: evaluation of the learning curve. Tech Coloproctol 2018; 22: 279-87.

26. Lee L, Kelly J, Nassif GJ, et al. Defining the learning curve for transanal total mesorectal excision for rectal adenocarcinoma. Surg Endosc 2018. doi: 10.1007/s00464-018-6360-4.

27. Mege D, Hain E, Lakkis Z, et al. Is trans-anal total mesorectal excision really safe and better than laparoscopic total mesorectal excision with a perineal approach first in patients with low rectal cancer? A learning curve with case-matched study in 68 patients. Colorectal Dis 2018; 20: 0143-51.
28. Persiani R, Biondi A, Pennestrì F, et al. Transanal total mesorectal excision vs laparoscopic total mesorectal excision in the treatment of low and middle rectal cancer: a propensity score matching analysis. Dis Colon Rectum 2018; 61: 809-16.

29. Lacy AM, Tasende MM, Delgado S, et al. Transanal total mesorectal excision for rectal cancer: outcomes after 140 patients. J Am Coll Surg 2015; 221: 415-23.

30. Martin-Perez B, Otero-Piñeiro A, Lacy AM. Purse-string rupture: pitfalls of transanal total mesorectal excision (Cecil approach). Tech Coloproctol 2018; 22: 393-4.

31. Araujo SE, Crawshaw B, Mendes CR, Delaney CP. Transanal total mesorectal excision: a systematic review of the experimental and clinical evidence. Tech Coloproctol 2015; 19: 69-82.

Received: 9.12.2018, accepted: 13.01.2019. 